\title{
KINEMATICS OF THE PLANAR QUADRILATERAL MECHANISM
}

\author{
Florian Ion Petrescu ${ }^{1}$ \\ Relly Victoria Petrescu ${ }^{2}$
}

\begin{abstract}
This paper presents an original method to determine the kinematic parameters at the linked quadrilateral mechanism. It is starting with a trigonometric method, which has the advantage to determine very quickly the position angles. The velocities can be determined faster using a geometric method. This method is developed and for the accelerations determinations. The (proposed) geometric method, determines first the kinematic parameters of the internal couple (B) and then the rotation angles with their derivatives. Secondary, the paper presents the determination of the efficiency of this mechanism. Determines and dynamic coefficient, D. With this one proposes two yields; the mechanical efficiency and the dynamic efficiency.
\end{abstract}

Keywords: 3R dyad, kinematic parameters, efficiency, dynamic coefficient

\section{INTRODUCTION}

The paper presents an original method to determine the kinematic parameters at the linked quadrilateral mechanism [3-4].

It is starting with a trigonometric method, which has the advantage to determine very quickly the position angles.

The velocities can be determined faster using a geometric method. This method is developed and for the accelerations determinations. The (proposed) geometric method, determines first the kinematic parameters of the internal couple (B) and then the rotation angles with their derivatives.

Secondary, the paper presents the determination of the efficiency of this mechanism. Determines and dynamic coefficient, $\mathrm{D}$.

With this one proposes two yields; the mechanical efficiency and the dynamic efficiency.

\section{THE KINEMATICS OF THE PLANAR QUADRILATERAL MECHANISM}

The kinematic schema of a planar quadrilateral mechanism [1-2] can be seen in the Figure 1.

The following kinematic parameters considered known:
$x_{O} ; y_{O} ; x_{C} ; y_{C} ; l_{1} ; l_{2} ; l_{3} ; \varphi_{1} ; \omega_{1}=c t . ;$ where the positions parameters $\left(x_{O} ; y_{O}\right)$ of the couple $\mathrm{O}$ are zero (if we set the rectangular system in $\mathrm{O}$ ); $1_{1}$ is the length of the crank $1 ; 1_{2}$ is the length of the connecting rod $2 ; 1_{3}$ is the length of the rocker 3; $\varphi_{1}$ is the crank angle position, and $\omega_{1}$ is its angular velocity. The $\mathrm{C}$ couple coordinates $\left(x_{C} ; y_{C}\right)$ are also known.

We must determine now the cinematic parameters which give the positions of the $\operatorname{rod}\left(\varphi_{2}\right)$ and the crank $\left(\varphi_{3}\right)$.

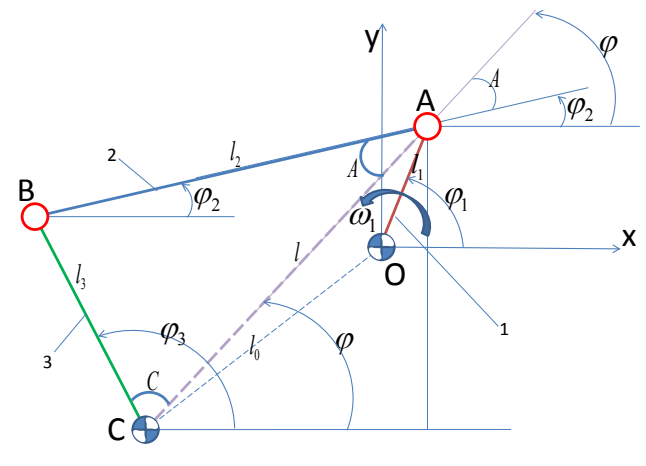

Fig. 1. Kinematic schema of a planar quadrilateral mechanism

\footnotetext{
1 Senior Lecturer Ph.D., Bucharest Polytechnic University, petrescuflorian@yahoo.com

2 Senior Lecturer Ph.D., Bucharest Polytechnic University, petrescuvictoria@yahoo.com
} 


$$
\begin{aligned}
& \left\{\begin{array} { l } 
{ x _ { A } = l _ { 1 } \cdot \operatorname { c o s } \varphi _ { 1 } } \\
{ y _ { A } = l _ { 1 } \cdot \operatorname { s i n } \varphi _ { 1 } }
\end{array} \quad \left\{\begin{array}{l}
\dot{x}_{A}=-l_{1} \cdot \sin \varphi_{1} \cdot \omega_{1} \\
\dot{y}_{A}=l_{1} \cdot \cos \varphi_{1} \cdot \omega_{1}
\end{array}\right.\right. \\
& \left\{\ddot{x}_{A}=-l_{1} \cdot \cos \varphi_{1} \cdot \omega_{1}^{2}\right. \\
& \ddot{y}_{A}=-l_{1} \cdot \sin \varphi_{1} \cdot \omega_{1}^{2} \\
& l^{2}=\left(x_{A}-x_{C}\right)^{2}+\left(y_{A}-y_{C}\right)^{2} \Rightarrow \\
& l=\sqrt{l^{2}}=\sqrt{\left(x_{A}-x_{C}\right)^{2}+\left(y_{A}-y_{C}\right)^{2}} \\
& \left\{\cos A=\frac{l^{2}+l_{2}^{2}-l_{3}^{2}}{2 \cdot l \cdot l_{2}} \Rightarrow A=\arccos (\cos A)\right. \text {; } \\
& \cos C=\frac{l^{2}+l_{3}^{2}-l_{2}^{2}}{2 \cdot l \cdot l_{3}} \Rightarrow C=\arccos (\cos C) \\
& \left\{\cos \varphi=\frac{x_{A}-x_{C}}{l}\right. \\
& \sin \varphi=\frac{y_{A}-y_{C}}{l} \\
& \Rightarrow \varphi=\operatorname{sign}(\sin \varphi) \cdot \arccos (\cos \varphi) ; \\
& \left\{\begin{array} { l } 
{ \varphi _ { 2 } = \varphi - A } \\
{ \varphi _ { 3 } = \varphi + C }
\end{array} \left\{\begin{array}{l}
x_{B}=x_{C}+l_{3} \cdot \cos \varphi_{3} \\
y_{B}=y_{C}+l_{3} \cdot \sin \varphi_{3}
\end{array}\right.\right.
\end{aligned}
$$

\section{DETERMINING THE POSITIONS}

It is starting with a trigonometric method, which has the advantage to determine very quickly the position angles (the system 1). First it determines the cinematic parameters of the $\mathrm{A}$ couple $\left(x_{A} ; y_{A}\right)$, and their first two derivatives ( $\left.\dot{x}_{A} ; \dot{y}_{A} ; \ddot{x}_{A} ; \ddot{y}_{A}\right)$. Second, one finds the variable length (l) between $\mathrm{C}$ and $\mathrm{A}$, and it determines the position angle $(\varphi)$ of the CA vector. We also determine the angles $\mathrm{A}$ and $\mathrm{C}$ from the triangle $\mathrm{ABC}$. Finally we found the positions of the rod ( $\left.\varphi_{2}\right)$ and the crank $\left(\varphi_{3}\right)$.

$$
\begin{aligned}
& \left\{\begin{array}{l}
\left(x_{B}-x_{C}\right)^{2}+\left(y_{B}-y_{C}\right)^{2}=l_{3}^{2} \\
\left(x_{B}-x_{A}\right)^{2}+\left(y_{B}-y_{A}\right)^{2}=l_{2}^{2}
\end{array} \Rightarrow\right. \\
& \Rightarrow\left\{\begin{array}{l}
\left(x_{B}-x_{C}\right) \cdot \dot{x}_{B}+\left(y_{B}-y_{C}\right) \cdot \dot{y}_{B}=0 \\
\left(x_{B}-x_{A}\right) \cdot \dot{x}_{B}+\left(y_{B}-y_{A}\right) \cdot \dot{y}_{B}= \\
=\left(x_{B}-x_{A}\right) \cdot \dot{x}_{A}+\left(y_{B}-y_{A}\right) \cdot \dot{y}_{A}
\end{array}\right. \\
& a_{11}=x_{B}-x_{C} ; \quad a_{12}=y_{B}-y_{C} ; \\
& a_{21}=x_{B}-x_{A} \\
& a_{22}=y_{B}-y_{A} ; \quad b_{1}=0 ; \\
& b_{2}=a_{21} \cdot \dot{x}_{\mathrm{A}}+a_{22} \cdot \dot{y}_{\mathrm{A}} \\
& \left\{\begin{array}{l}
\left\{\begin{array}{l}
a_{11} \cdot \dot{\mathrm{x}}_{B}+a_{12} \cdot \dot{y}_{B}=b_{1} \\
a_{21} \cdot \dot{\mathrm{x}}_{B}+a_{22} \cdot \dot{\mathrm{y}}_{B}=b_{2}
\end{array} \Rightarrow\right. \\
\Delta=\left|\begin{array}{ll}
a_{11} & a_{12} \\
a_{21} & a_{22}
\end{array}\right|=a_{11} \cdot a_{22}-a_{12} \cdot a_{21}
\end{array} ;\right. \\
& \Delta_{\dot{x}_{B}}=\left|\begin{array}{ll}
b_{1} & a_{12} \\
b_{2} & a_{22}
\end{array}\right|=b_{1} \cdot a_{22}-a_{12} \cdot b_{2} \\
& \Delta_{\dot{y}_{B}}=\left|\begin{array}{ll}
a_{11} & b_{1} \\
a_{21} & b_{2}
\end{array}\right|=a_{11} \cdot b_{2}-a_{21} \cdot b_{1} ; \\
& \dot{x}_{B}=\frac{\Delta_{\dot{x}_{B}}}{\Delta} ; \quad \dot{y}_{B}=\frac{\Delta_{\dot{y}_{B}}}{\Delta}
\end{aligned}
$$

\section{DETERMINING THE VELOCITIES OF THE COUPLE B}

The velocities $\left(\dot{x}_{B} ; \dot{y}_{B}\right)$ can be determined faster using a geometric method (the system 2). First, we write the equations of the circles which have the radius $\mathrm{l}_{3}$ and $\mathrm{l}_{2}$. They intersect in $\mathrm{B}$. This system does not have to be solve because the solutions are already known. It will be derivative two times to obtain the systems of velocity and accelerations. These systems are linear and are solved by determinants. At 2.2 we obtain the velocities ( $\dot{x}_{B} ; \dot{y}_{B}$ ), and at 2.3 determine the accelerations ( $\left.\ddot{x}_{B} ; \ddot{y}_{B}\right)$.

\section{DETERMINING THE ACCELERATIONS OF THE COUPLE B}


The accelerations $\left(\ddot{x}_{B} ; \ddot{y}_{B}\right)$ can be determined faster using a geometric method (the system 3).

$$
\left\{\begin{array}{l}
\left\{\begin{array}{l}
\left(x_{B}-x_{C}\right) \cdot \ddot{x}_{B}+\left(y_{B}-y_{C}\right) \cdot \ddot{y}_{B}= \\
=-\dot{x}_{B}^{2}-\dot{y}_{B}^{2} \\
\left(x_{B}-x_{A}\right) \cdot \ddot{x}_{B}+\left(y_{B}-y_{A}\right) \cdot \ddot{y}_{B}= \\
=a_{21} \cdot \ddot{x}_{A}+a_{22} \cdot \ddot{y}_{A}-\dot{a}_{21}^{2}-\dot{a}_{22}^{2}
\end{array}\right. \\
c_{1}=-\dot{x}_{B}^{2}-\dot{y}_{B}^{2} ; \\
c_{2}=a_{21} \cdot \ddot{x}_{A}+a_{22} \cdot \ddot{y}_{A}-\dot{a}_{21}^{2}-\dot{a}_{22}^{2} \\
\left\{\begin{array}{l}
a_{11} \cdot \ddot{x}_{B}+a_{12} \cdot \ddot{y}_{B}=c_{1} \\
a_{21} \cdot \ddot{x}_{B}+a_{22} \cdot \ddot{y}_{B}=c_{2}
\end{array}\right. \\
\Delta_{\ddot{x}_{B}}=\left|\begin{array}{ll}
c_{1} & a_{12} \\
c_{2} & a_{22}
\end{array}\right|=c_{1} \cdot a_{22}-a_{12} \cdot c_{2} \\
\Delta_{\ddot{y}_{B}}=\left|\begin{array}{ll}
a_{11} & c_{1} \\
a_{21} & c_{2}
\end{array}\right|=a_{11} \cdot c_{2}-a_{21} \cdot c_{1}
\end{array}\right.
$$

\section{DETERMINING THE ANGULAR VELOCITIES AND ACCELERATIONS}

The angular velocities $\left(\omega_{2} ; \omega_{3}\right)$ and accelerations $\left(\varepsilon_{2} ; \varepsilon_{3}\right)$ can be determined now, faster, using the vectorial method (system 4 ).

$$
\left\{\begin{array}{l}
\left\{\begin{array}{l}
\left\{\begin{array}{l}
x_{A}-x_{B}=l_{2} \cdot \cos \varphi_{2} \\
y_{A}-y_{B}=l_{2} \cdot \sin \varphi_{2}
\end{array}\right. \\
\left\{\begin{array}{l}
\dot{x}_{A}-\dot{x}_{B}=-l_{2} \cdot \sin \varphi_{2} \cdot \omega_{2} \mid \cdot\left(-\sin \varphi_{2}\right) \\
\dot{y}_{A}-\dot{y}_{B}=l_{2} \cdot \cos \varphi_{2} \cdot \omega_{2} \mid \cdot\left(\cos \varphi_{2}\right) \\
\Rightarrow \omega_{2}=\frac{\left(\dot{y}_{A}-\dot{y}_{B}\right) \cdot \cos \varphi_{2}-\left(\dot{x}_{A}-\dot{x}_{B}\right) \cdot \sin \varphi_{2}}{l_{2}}
\end{array}\right. \\
\left\{\begin{array}{l}
\ddot{x}_{A}-\ddot{x}_{B}=-l_{2} \cdot \sin \varphi_{2} \cdot \varepsilon_{2}-l_{2} \cdot \cos \varphi_{2} \cdot \omega_{2}^{2} \mid \cdot\left(-\sin \varphi_{2}\right) \\
\ddot{y}_{A}-\ddot{y}_{B}=l_{2} \cdot \cos \varphi_{2} \cdot \varepsilon_{2}-l_{2} \cdot \sin \varphi_{2} \cdot \omega_{2}^{2} \mid \cdot\left(\cos \varphi_{2}\right)
\end{array} \Rightarrow\right. \\
\Rightarrow \begin{array}{l}
\varepsilon_{2}=\frac{\left(\ddot{y}_{A}-\ddot{y}_{B}\right) \cdot \cos \varphi_{2}-\left(\ddot{x}_{A}-\ddot{x}_{B}\right) \cdot \sin \varphi_{2}}{l_{2}} \\
\left\{\begin{array}{l}
x_{B}-x_{C}=l_{3} \cdot \cos \varphi_{3} \\
y_{B}-y_{C}=l_{3} \cdot \sin \varphi_{3} \\
\dot{x}_{B}-\dot{x}_{C}=-l_{3} \cdot \sin \varphi_{3} \cdot \omega_{3} \mid \cdot\left(-\sin \varphi_{3}\right)
\end{array} \Rightarrow \dot{y}_{C}=l_{3} \cdot \cos \varphi_{3} \cdot \omega_{3} \mid \cdot\left(\cos \varphi_{3}\right)\right.
\end{array} \Rightarrow \\
\Rightarrow \omega_{3}=\frac{\left(\dot{y}_{B}-\dot{y}_{C}\right) \cdot \cos \varphi_{3}-\left(\dot{x}_{B}-\dot{x}_{C}\right) \cdot \sin \varphi_{3}}{l_{3}} \\
\left\{\begin{array}{l}
\ddot{x}_{B}-\ddot{x}_{C}=-l_{3} \cdot \sin \varphi_{3} \cdot \varepsilon_{3}-l_{3} \cdot \cos \varphi_{3} \cdot \omega_{3}^{2} \mid \cdot\left(-\sin \varphi_{3}\right) \\
\ddot{y}_{B}-\ddot{y}_{C}=l_{3} \cdot \cos \varphi_{3} \cdot \varepsilon_{3}-l_{3} \cdot \sin \varphi_{3} \cdot \omega_{3}^{2} \mid \cdot\left(\cos \varphi_{3}\right)
\end{array} \Rightarrow\right. \\
\Rightarrow \varepsilon_{3}=\frac{\left(\ddot{y}_{B}-\ddot{y}_{C}\right) \cdot \cos \varphi_{3}-\left(\ddot{x}_{B}-\ddot{x}_{C}\right) \cdot \sin \varphi_{3}}{l_{3}}
\end{array} \Rightarrow\right.
\end{array}\right.
$$

\section{THE EFFICIENCY OF THE PLANAR QUADRILATERAL MECHANISM}

The efficiency of a planar quadrilateral mechanism can be determined starting from the forces and velocities repartition, (Figure 2).

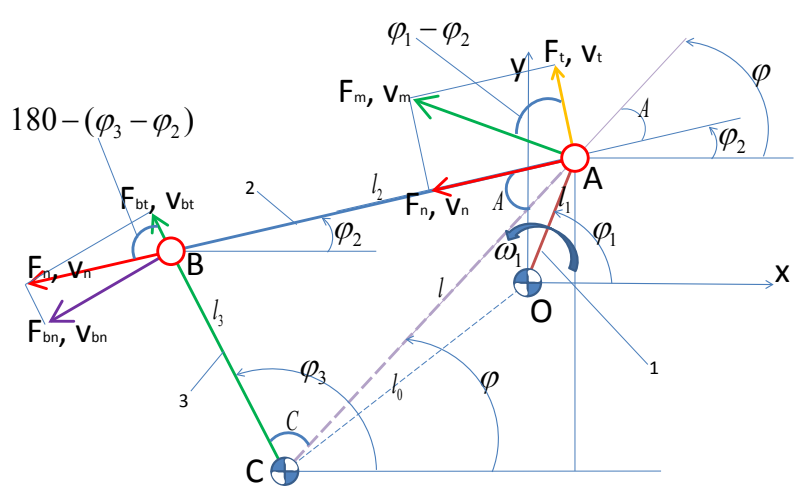

Fig. 2. Forces and velocities repartition of a planar quadrilateral mechanism

The system (5) presents the relationships which give the forces and the velocities on the planar quadrilateral mechanism. The driving force $\mathrm{F}_{\mathrm{m}}$ is perpendicular on the crank 1 in $\mathrm{A}$.

Its component along the connecting rod 
(the bar 2) $F_{n}$, gives the normal component $F_{b n}$. $\mathrm{F}_{\mathrm{bn}}$ is perpendicular on the rocker 3 in $\mathrm{B}$.

These forces give the dynamic velocities which are similar with the forces.

The forces are always the same, but the velocities (the dynamic velocities) are different than the kinematics velocities.

For this reason the dynamic efficiency will be different than the mechanical yield.

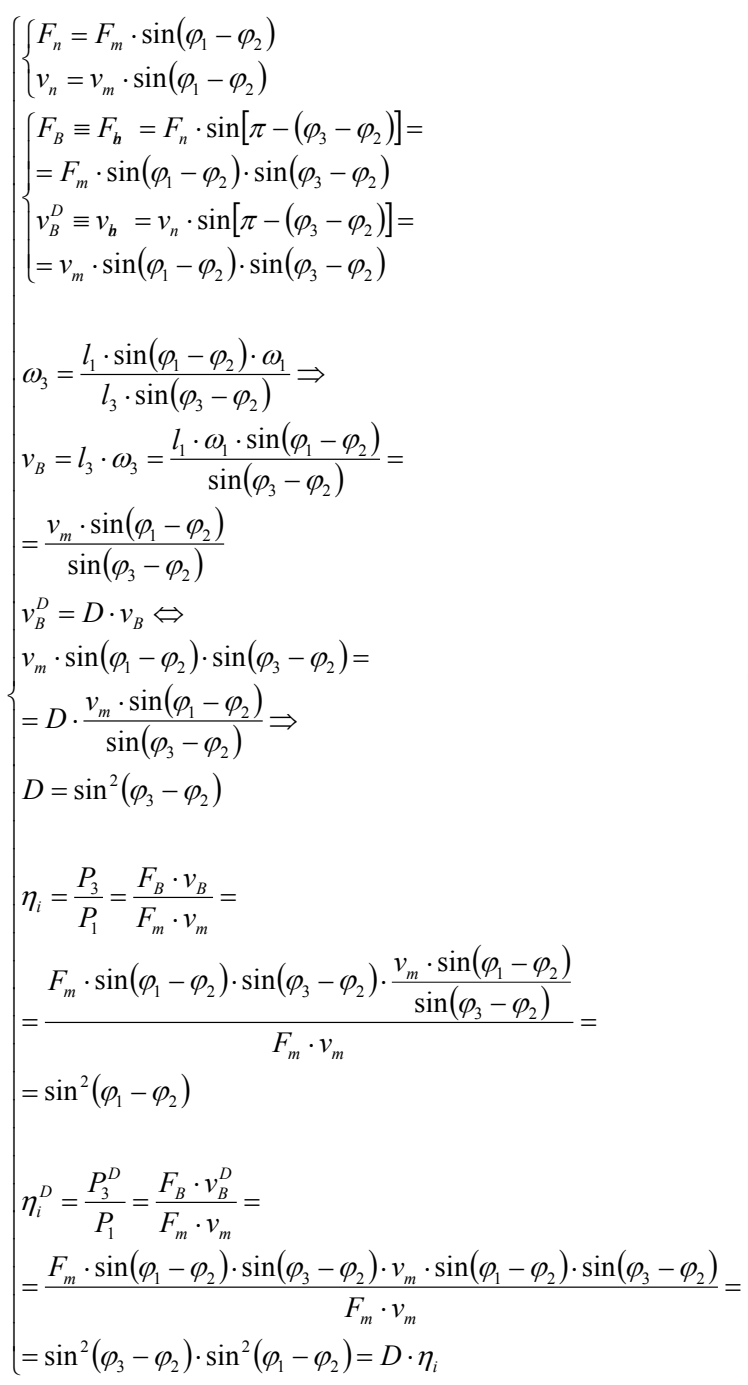

\section{CONCLUSIONS}

The presented method is the most elegant and direct method to determine the kinematics planar quadrilateral mechanism.

Relationships used by this method allow and the determination of the dynamic system vibration. In the dynamic kinematics the constant rotation speed $\omega_{1}=\mathfrak{c}$. gets a variable value
$\omega_{1}^{D}=D \cdot \omega_{1}$. We consider that the rotation speed $\left(\omega_{1}\right)$ of the crank is a constant for a constant rotation speed (n) (relation 6).

$$
\omega_{1}=2 \cdot \pi \cdot v=2 \cdot \pi \cdot \frac{\dot{n}}{60}=\frac{\pi \cdot n}{30}
$$

In fact $\omega_{1}$ varies with the position of the crank angle $(\varphi)$ (system 7, [3-4]).

$$
\begin{aligned}
& \left\{\begin{array}{l}
\omega_{1 m}=D \cdot \frac{\pi \cdot n}{30}=\sin ^{2}\left(\varphi_{3}-\varphi_{2}\right) \cdot \frac{\pi \cdot n}{30} ; \\
J_{m}^{*}=J_{\text {medium }}^{*}=\frac{J_{\min }^{*}+J_{\max }^{*}}{2} ; \\
\omega_{1}=\omega_{1 m} \cdot \sqrt{\frac{J_{m}^{*}}{J^{*}}}= \\
=\sin ^{2}\left(\varphi_{3}-\varphi_{2}\right) \cdot \frac{\pi \cdot n}{30} \cdot \sqrt{\frac{J_{m}^{*}}{J^{*}}} \\
\varepsilon_{1}=-\frac{\omega_{1}^{2}}{2} \cdot \frac{J^{*}}{J^{*}}=-\frac{\omega_{1}^{2}}{2} \cdot \frac{d J^{*}}{J^{*}} \\
\quad J^{*} \text { is the mechanical moment of inertia re- }
\end{array}\right. \\
& \text { duced to crank; } J_{m}^{*}=J_{\text {medium }}^{*} .
\end{aligned}
$$

\section{REFERENCES}

Erdman, A.G., Three and four precision point kinematic synthesis of planar linkages, Elsevier, Mechanism and Machine Theory, Volume 16, Issue 3, 1981, pages $227-245$.

Gibson, C.G., Newstead, P.E., On the geometry of the planar 4-bar mechanism, Acta Applicandae Mathematicae, Volume 7, Number 2, pages 113135.

Petrescu, F.I., Teoria Mecanismelor si a Masinilor, Create Space Publisher, USA, 2011, ISBN/EAN 13: 1468015826 / 978-1-4680-1582-9, page count 432 .

Petrescu, F.I., Bazele Analizei si Optimizarii Sistemelor cu Memorie Rigida, Create Space Publisher, USA, 2012, ISBN/EAN 13: 1470024365 / 9781-4700-2436-9, page count 164. 